\title{
Neurotrophic Activity in the Adult Rat Hippocampal Formation: Regional Distribution and Increase after Septal Lesion'
}

\author{
FRANK COLLINS ${ }^{2}$ AND KEITH A. CRUTCHER
}

\author{
Department of Anatomy, University of Utah School of Medicine, Salt Lake City, Utah 84132
}

\begin{abstract}
Conditioned medium prepared from slices of the rat hippocampal formation contains an agent that shares the following properties with nerve growth factor (NGF): (1) it promotes neurite growth from embryonic sympathetic ganglia in vitro; and (2) it is inhibited by affinity-purified antibody against mouse submaxillary gland $2.5 \mathrm{~S}$ NGF. The NGF-like growthpromoting activity is regionally distributed within the hippocampal formation: the activity is consistently higher in the dentate gyrus-CA3 region than in the CA1 region. Furthermore, the level of activity is significantly increased within 1 week after a medial septal lesion, and, in the dentate-CA3 region, the increased level of activity is maintained for at least 4 weeks after the lesion. Control lesions that fail to interrupt the septohippocampal innervation, but cause equally extensive damage to nearby regions of the central nervous system, do not cause increased levels of activity in the hippocampal formation. These results provide substantial evidence linking the NGF-like agent in hippocampal conditioned medium to the sprouting of sympathetic axons into the dentate gyrus-CA3 region of the hippocampal formation after a medial septal lesion in vivo.
\end{abstract}

Neuronal development and the neuronal rearrangements that follow injury to the adult nervous system both involve new neurite growth. Several lines of evidence implicate a number of different neuronal growth factors in the regulation of neurite growth during development (Berg, 1984; Collins, 1984a). Much less evidence is available regarding the role of the same or analogous factors in neuronal growth following injury to the adult nervous system. The sprouting of sympathetic fibers into the hippocampal formation following damage to the septal innervation in adult rats is a useful model for studying the factors that regulate neuronal rearrangements following injury (Crutcher and Davis, 1981). The sympathetic ingrowth is localized to very specific regions within the hippocampal formation, suggesting that the responsc to injury in this instance is precisely controlled. A number of studies of this system have suggested that sympathetic fibers may be induced to grow, and are directed in their growth, by a neuronal growth factor emanating from the specific regions of the hippocampal formation in which the

Received January 7, 1985; Revised March 15, 1985;

Accepted March 15, 1985

\footnotetext{
${ }^{1}$ This work was supported by the excellent technical assistance of Margaret Vogel and Yvonne Mueller, and by Grant NS-17131 from the National Institutes of Health.

${ }^{2}$ To whom correspondence should be addressed, at Department of Anatomy, Room 435, Wintrobe Building, University of Utah School of Medicine, Salt Lake City, UT 84132.
}

sympathetic fibers become localized, the dentate gyrus and CA3 regions. This putative hippocampal growth factor has been compared to nerve growth factor (NGF), based on its ability to induce and direct the growth of sympathetic axons (Crutcher et al., 1979, 1981). A key corollary of this hypothesis is that damage to the septal innervation of the hippocampal formation should result in sufficiently increased levels of this growth factor to induce sympathetic ingrowth.

We reported previously that hippocampal homogenates containcd an agent with biological and antigenic similarities to mouse submaxillary gland NGF (Crutcher and Collins, 1982). The present experiments confirm these results, using hippocampal conditioned medium instead of homogenates, an assay system based on undissociated sympathetic ganglia instead of dissociated neurons, and affinitypurified anti-NGF antibody instead of unfractionated antiserum. However, the more immediate purpose of the present experiments was to determine whether a medial septal lesion, known to damage the septohippocampal innervation, would result in a sustained increase in NGF-like activity within those regions of the hippocampal formation that receive the sympathetic ingrowth after a medial septal lesion in vivo.

\section{Materials and Methods}

Culturc conditions. Culturc medium consistcd of Ham's F12 supplo mented with $5 \%$ heat-inactivated horse serum. Cultures were incubated at $37^{\circ} \mathrm{C}$ and $98 \%$ humidity with sufficient $\mathrm{CO}_{2}$ to maintain the $\mathrm{pH}$ between 7.2 and 7.4. All dissections and culture manipulations were performed in a laminar flow hood.

Hippocampal conditioned medium. Adult female albino rats were used. Animals were decapitated and the brain was removed. The hippocampal formation was dissected from the brain bilaterally and placed with the alveus facing down on the stage of a dissecting microscope in a dish containing culture medium. The septal (left hippocampal formation) or temporal (right hippocampal formation) pole was fastened in place with a dissecting pin and transverse slices, 1 to $2 \mathrm{~mm}$ in thickness, were cut with iridectomy knives beginning at the pole opposite the pin. The orientation of the hippocampal formation allowed for slices to be made from the medial to lateral direction, ending at the fimbrial side. This minimized the distortion of the tissue during slicing. A total of 8 or 9 slices were obtained from each hippocampal formation (16 to 18 per animal). After trimming away excess fimbrial or subicular tissue, each slice was dissected into CA1 and dentate-CA3 portions. The division between CA1 and the dentate was made along the hippocampal fissure, and this plane was extended to bisect the transition zone (CA2) between CA1 and CA3. Each slice was transferred to a $6.4-\mathrm{mm}$ tissue culture microwell containing $0.15 \mathrm{ml}$ of culture medium. After $48 \mathrm{hr}$ incubation, all of the media containing the CA1 stices of one animal were pooled (about $2.5 \mathrm{ml}$ total), as were the media containing the dentate-CA3 slices. Conditioned media were centrifuged at $15,000 \times \mathrm{g}$ for $15 \mathrm{~min}$. The supernatants were collected and assayed immediately. After removal of the medium, hippocampal slices were air dried and weighed. Occasionally, individual slices were fixed in $4 \%$ paraformaldehyde, dehydrated, and embedded in paraffin for histological analysis to determine the accuracy of the dissection. Histological analysis revealed that, although there were some CA1 slices that included small portions of CA3 and the subiculum, the 
dissection produced a consistent separation of slices containing granule cells and their axons (i.e., dentate-CA3) and slices without granule cells (i.e., CA1).

Other conditioned media and tissue extracts. Heart cell conditioned medium was prepared from confluent monolayer cultures of embryonic day 10 (E10) chick embryo heart cells, as previously described (Collins, 1978a). Parietal yolk sac conditioned medium (PYSCM) was prepared from confluent monolayers of a mouse parietal yolk sac endoderm cell line (Lehman et al., 1974), as previously described (Collins, 1984b). Extract of chick embryo (E16) eye tissues was prepared as described by Adler et al. (1979).

Assay of neurite-stimulating activity. Plastic $35-\mathrm{mm}$ tissue culture dishes were pretreated with polyornithine and PYSCM (see above) in order to prepare the culture substratum for neurite growth (Collins, 1978b, 1984b). Each hippocampal conditioned medium was assayed in two duplicate dishes, with cach dish rocciving $1.5 \mathrm{ml}$ of modium consisting of cqual parts hippo campal conditioned medium and unconditioned medium. The lumbar sympathetic chain was dissected from E9 White Leghorn chick embryos, freed of surrounding tissues, and cul into approximalely 1 -mm pieces. Eight to 10 pieces of sympathetic chain were added to each dish. After $48 \mathrm{hr}$ incubation, dishes were placed on the stage of a phase contrast microscope to measure neurite growth. Une edge of an eyepiece grid reticle was visually aligned with the edge of each ganglionic explant at four orthogonally directed sites around the explant. The number of neurites crossing the other side of the reticle, $400 \mu \mathrm{m}$ from the explant, was counted and averaged over the four locations for each explant. The value so obtained for each of the 10 explants was averaged for each culture to obtain the neurite index (for details, see Collins, 1984b). The neurite index for each conditioned medium was then divided by the average dry weight (in milligrams) per slice of tissue used to prepare the conditioned medium, in order to obtain the specific activity neurite index plottcd under "Rcsults." The statistical significance of the difference in neurite index for different experimental treatments was determined using the Mann-Whitney $U$ test. Except as noted, all of the results reported here were obtained using aliquots of the same batch of PYSCM to pretreat the culture substratum. When other batches, or different dilutions of the same batch, were used, results similar to those reported here were consistently obtained (see Table 1), but the absolute values of the neurite index were different.

Lesions. Electrolytic lesions were placed in the septal area or bilaterally in the cortex overlying the hippocampal formation. The lesion site was located stereotaxically and $3 \mathrm{~mA}$ of direct current were delivered for $15 \mathrm{sec}$, as previously described (Crutcher et al., 1981). When animals were later sacrificed, the appropriate area of the brain was frozen and sectioned to determine microscopically the size and location of the lesion.

NGF and anti-NGF antibody. Affinity-purified anti-mouse submaxillary gland 2.5 S NGF, obtained from Laref, SA (Cadempino-TI, Switzerland), was used at a final concentration of $5 \mu \mathrm{g} / \mathrm{ml}$. Antibody was added to the medium for $30 \mathrm{~min}$ before addition of sympathetic chain pieces. Purified mouse submaxillary gland $\beta$-NGF was purchased from The Research Foundation of the State University of New York (Albany, NY).

\section{Results}

Culture medium conditioned by exposure for $48 \mathrm{hr}$ to tissue slices from the rat hippocampal formation dramatically increased the extent of neurite growth from ganglia of the chick embryo sympathetic chain in vitro (Fig. 1). In unconditioned culture medium the extent of sympathetic neurite growth (neurite index) was below the minimum level of the assay.

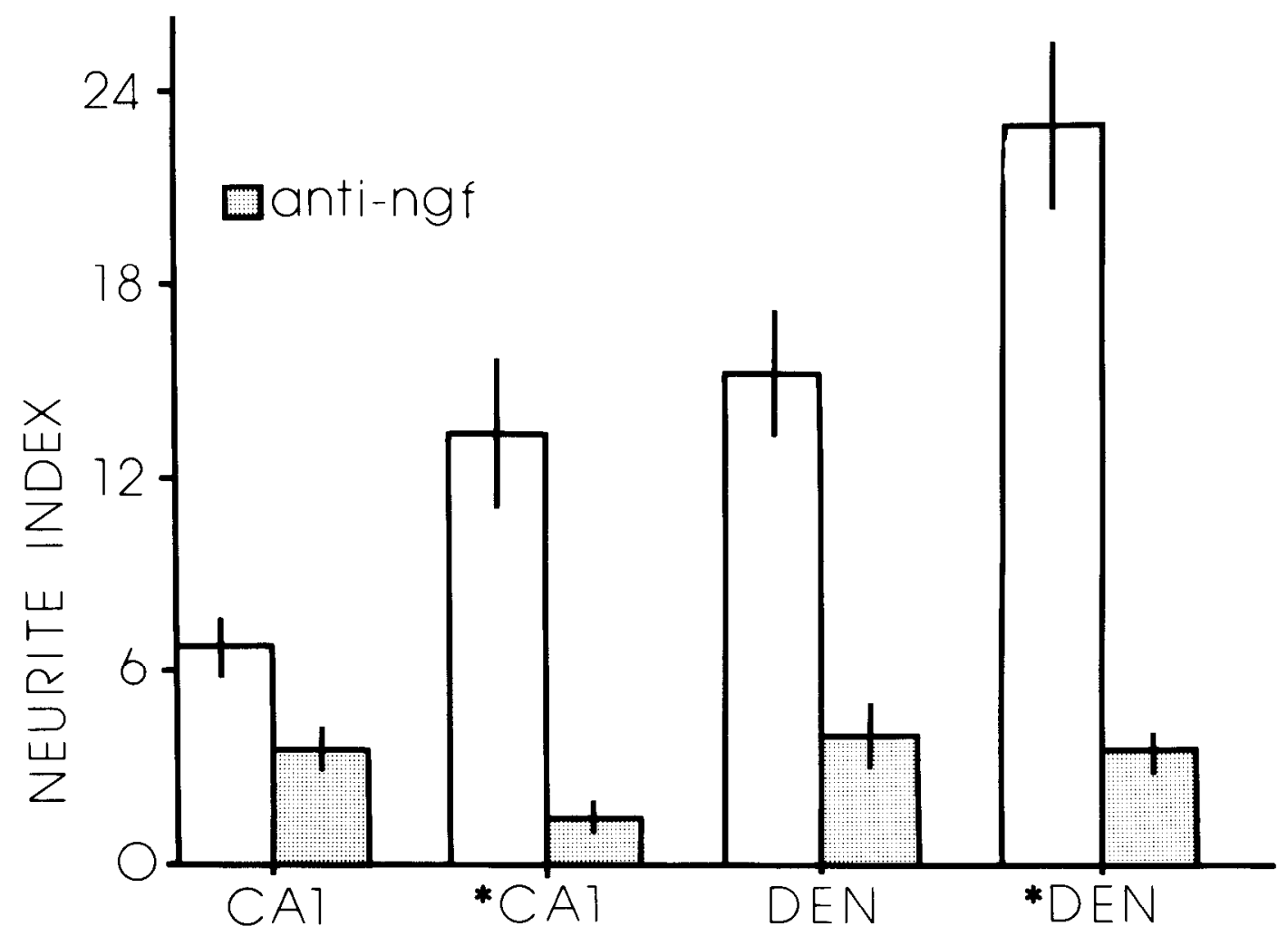

Figure 1. Effect of septal lesion and of antibodies against $2.5 \mathrm{~S} \mathrm{NGF}$ on the stimulation of sympathetic neurite growth by hippocampal conditioned medium. The hippocampal formation was removed bilaterally frorn rats that either had received a septal lesion 1 week earlier $(*)$ or were unlesioned controls. Separate conditioned media were prepared from slices of the CA1 and of the dentate-CA3 (DEN) regions of each animal. Pieces of chick embryo sympathetic chain were cultured in these conditioned media for 2 days, at which time the extent of neurite growth (NEURITE INDEX) was measured. The neurite index represents a specific activity since it has been corrected for the dry weight of hippocampal tissue used to prepare the conditioned medium, as described under "Materials and Methods." The left bar of each pair of bars represents the mean value of the neurite index \pm the standard deviation for at least 11 such experiments. In 3 experiments of this series, an aliquot of each conditioned medium was also exposed to $5 \mu \mathrm{g} / \mathrm{ml}$ of affinity-purified anti-NGF before addition to the sympathetic chain cultures. The mean neurite index \pm the standard deviation for the anti-NGF-treated conditioned medium is shown as the right bar of each pair. In all of these experiments the neurite index of sympathetic chain cultures incubated in unconditioned medium was 0 . The statistical significance of the results was as follows: (1) the difference between conditioned medium treated with antibody and without was highly statistically significant $(\rho<$ 0.001 , except for unlesioned CA1, where $p<0.01$ ); and (2) the difference between conditioned medium from lesioned and unlesioned animals was also highly significant $(p<0.001)$. 
The effectiveness of hippocampal conditioned medium in stimulating sympathetic neurite growth depended both upon the region of the hippocampal formation used to condition the medium and upon whether or not the animal had received a prior medial septal lesion. Conditioned medium from the dentate-CA3 region stimulated sympathetic neurite growth at least 2 -fold more than did conditioned medium from the CA1 region (Fig. 1). The effectiveness of conditioned medium from both regions was increased an additional 1.5to 2-fold by medial septal lesion 1 week before the preparation of hippocampal conditioned medium (Fig. 1, asterisks).

When the survival period following the medial septal lesion was increased to 4 weeks, the neurite-stimulating activity in dentate-CA3 conditioned medium remained at approximately the same elevated level as at 1 week after the lesion (Fig. 2). In contrast, the activity in CA1 conditioned medium, prepared 4 weeks after a septal lesion, had decreased to about the level of the unlesioned controls (Fig. 2). These results indicate that after a medial septal lesion, (1) the neurite-stimulating activity is significantly increased in both dentateCA 3 and CA1 within 1 week, (2) the increased activity after the lesion is maintained for at least 4 weeks in dentate-CA3 but not $\mathrm{CA} 1$, and (3) the absolute level of activity is significantly higher in dentate-CA3 than in CA1.

The lesion data described above were from animals that had sustained a medial septal lesion comparable to those which have previously been shown to elicit sympathetic sprouting into the hippocampal formation in vivo (Stenevi and Bjorklund, 1978; Crutcher et al., 1979, 1981). In additional experiments, two types of control lesion were given in order to test the hypothesis that the increase in neurite-promoting activily after medial septal lesion was related to denervation of the hippocampal formation and not to nonspecific effects of tissue damage in nearby regions of the CNS. One type of control lesion caused approximately the same tissue damage as the medial septal lesion but was applied immediately caudal to the normal location of a medial septal lesion, in a position unlikely to have damaged the septohippocampal innervation. The second type of lesion was applied much closer to the hippocampal formation, in the overlying cerebral cortex. This lesion was applied bilaterally so that the amount of tissue damage was approximately twice that resulting from a medial septal lesion, but the septohippocampal pathway was left intact. Both of these types of control lesion failed to cause a significant increase above intact controls in the levels of neurite-promoting activity in hippocampal conditioned medium from either the dentate-CA3 or CA1 regions (Table I). The control losion cxperiments were done under conditions identical to those in which a medial septal lesion invariably caused elevated levels of neurite-promoting activity (Fig. 1). When animals that had received a medial septal lesion were included in these experiments as positive controls, they exhibited the typical increase in activity above the levels in intact animals (Table I).

The stimulation of neurite growth by hippocampal conditioned medium was significantly reduced by prior exposure of the medium to affinity-purified antibody against mouse submaxillary gland $2.5 \mathrm{~S}$ NGF (Fig. 1). Since the amount of activity remaining in the presence of anti-NGF was approximately the same for both hippocampal regions, with and without prior medial septal lesion (Fig. 1), the actual percentage of inhibition depended on the starting activity. For

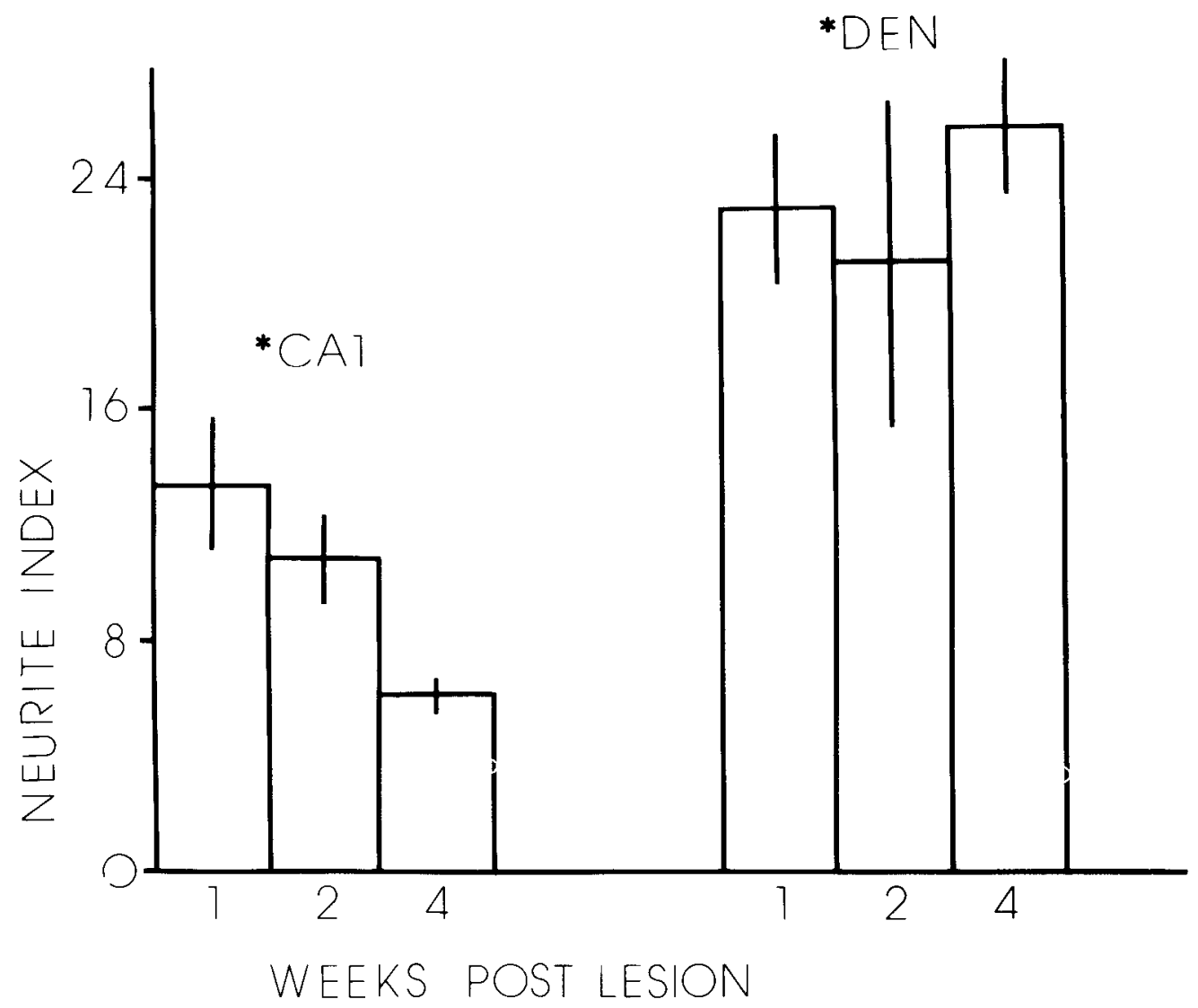

Figure 2. Effect of time after lesion on the stimulation of sympathetic neurite growth by CA1 and dentate conditioned medium. Conditioned media were prepared from the CA1 and from the dentate-CA3 $(D E N)$ regions of the hippocampal formation of rats that had received a septal lesion 1,2 , or 4 weeks earlier. The extent of neurite growth (NEURITE INDEX) in these various conditioned media was measured and corrected for the dry weight of hippocampal tissue as described in the legend to Figure 1. Each bar represents the mean neurite index \pm the standard deviation. The 1 week data are the same as those plotted in Figure 1, whereas the 2 and 4 week data are each from two additional experiments. There is no statistical significance between the 1,2 and 4 week values for the dentate-CA3 region of lesioned animals $(p>0.25)$. For the CA1 region, the difference between the values at 1 or 2 weeks and the values at 4 weeks is highly significant $(p<0.001)$ 


\section{TABLE I}

Effect of control lesions on the stimulation of sympathetic neurite growth by hippocampal conditioned medium

Conditioned medium was prepared from the CA1 and dentate-CA3 (DEN) regions of hippocampal formations taken from rats that either had received no septal lesion, a medial septal lesion, or a control lesion consisting either of a caudal septal lesion or a bilateral cortical lesion, both of which failed to denervate the hippocampus. Animals were sacrificed 1 week after lesion. The extent of sympathetic neurite growth (Neurite Index) in these conditioned media was measured as described in the legend to Figure 1 . The data in the top half of the table are from the same series of experiments reported in Figures 1 and 2. The data in the bottom half are from other experiments using a different pretreatment of the culture substratum, which resulted in different absolute values of the neurite index but the same relative increases after medial septal lesion (see "Materials and Methods").

\begin{tabular}{clc}
\hline Hippocampal Region & \multicolumn{1}{c}{ Lesion } & Neurite Index \\
\hline CA1 & None & 7.2 \\
CA1 & Caudal & 7.8 \\
DEN & None & 18.0 \\
DEN & Caudal & 17.9 \\
CA1 & None & 2.3 \\
CA1 & Cortical & 3.2 \\
CA1 & Medial septal & 6.0 \\
DEN & None & 8.4 \\
DEN & Cortical & 8.7 \\
DEN & Medial septal & 17.0 \\
\hline
\end{tabular}

the particular case of conditioned medium from the dentate-CA3 region of animals that had received a medial septal lesion, anti-NGF caused more than an $80 \%$ inhibition of the neurite index (Fig. 1).

The following experiments were performed to control for a possible nonspecific inhibitory effect of the anti-NGF antibody. In place of hippocampal conditioned medium, sympathetic chain neurite growth was stimulated by a number of conditioned media and tissue extracts the effectiveness of which is not due to the presence of NGF (Helfand et al., 1978; Collins and Dawson, 1983; Collins, 1984b). The anti-NGF anlibody failed to inhibit significantly the stimulation of neurite growth by these conditioned media and tissue extracts, as shown in Figure 3. Also shown in Figure 3 is the fact that the anti-NGF antibody completely inhibited the stimulation of sympathetic neurite growth by mouse submaxillary gland $\beta$-NGF. Together these results suggest that the affinity-purified anti-NGF antibody antagonized an NGF-like component in the hippocampal conditioned media, rather than directly inhibiting neurite growth.

\section{Discussion}

A series of in vivo experiments has demonstrated that a specific denervation of the hippocampal formation causes the ingrowth of sympathetic axons. Loss of the septal cholinergic innervation to the hippocampal formation, resulting from a medial septal lesion, or fimbrial transection, causes sympathetic axons associated with nearby blood vessels to sprout and grow into the dentate-CA3 region of the hippocampal formation (Loy and Moore, 1977; Stenevi and Bjorklund, 1978; Crutcher et al., 1979, 1981; Loy et al., 1980). The restricted distribution of sympathetic fibers to dentate-CA3 appears to correlate with the distribution of dentate granule cells and their axons, the mossy fibers (Crutcher and Davis, 1982). There is little, if any, sympathetic ingrowth into the CA1 region of the hippocampal formation, an area that receives a sparse septal cholinergic innervation and lacks mossy fibers. Sympathetic axons invade the dentate-CA3 region in essentially the same way that they would invade a region of the CNS that had received a local injection of NGF (Levi-Montalcini et al., 1978). These observations led to the proposal that the dentate-CA3 region of the hippocampal formation contains an endogenous NGF-like agent, the local concentration of which is down regulated by the septal fibers. Destruction of these fibers would result in a sufficiently elevated concentration to induce sympathetic sprouting (Crutcher et al., 1979). This hypothesis caused us to examine the hippocampal formation for the presence of such an NGF-like agent: one in which the growth-promoting effects on sympathetic neurons, regional distribution within the hippocampal formation, and elevation after a medial septal lesion corresponded to the pattern predicted from the in vivo experiments. We believe that the present results, together with previous observations (Crutcher and Collins, 1982), provide evidence for the existence of such a factor.

The present results, along with those obtained earlier, indicate that the hippocampal formation contains an agent that resembles NGF in two ways. The first similarity is the ability to stimulate neurite growth from embryonic sympathetic nerve cells in both undissociated ganglia (Figs. 1 and 2, Table I) and dissociated neuronal cultures (Crutcher and Collins, 1982). The second sirmilarity is inhibition of the activity by affinity-purified antibody against mouse submaxillary gland 2.5 S NGF (Fig. 1) and by anti-NGF antiserum (Crutcher and Collins, 1982). Control experiments (Fig. 3; Crutcher and Collins, 1982) confirm that the anti-NGF antibody is acting selectively on the hippocampal agent and NGF, rather than nonspecifically inhibiting neurite growth. It has also been reported that the denervated hippocampal formation exerts an NGF-like effect on transplanted sympathetic ganglia in vivo (Bjorklund and Stenevi, 1981; Gage et al., 1984). Although the hippocampal formation apparently contains an agent with biological and antigenic similarities to NGF, it would be incorrect to conclude that the hippocampal agent is identical to mouse submaxillary gland NGF.

The present experiments also extend these findings by demonstrating (1) that the neurite-stimulating activity is consistently higher in the dentate-CA3 region than in the CA1 region, and (2) that after a medial septal lesion the activity is substantially elevated for an extended period in dentate-CA3 but not CA1. This elevation in levels of activity after lesion is not observed when the lesion fails to interrupt the septal innervation to the hippocampus (Table I), even though equally extensive damage to areas adjacent to the scptum or hippocampal formation has occurred. Such lesions are also known not to elicit sympathetic sprouting in vivo (Crutcher et al., 1981). Also, the maximal increase in NGF-like activity occurs by 9 days after a medial septal lesion (Fig. 2), which is the time when sympathetic ingrowth can first be detected (Milner and Loy, 1980). These additional findings strengthen the argument that the neurite-stimulating activity observed in vitro has the properties expected of the agent responsible for sympathetic sprouting after medial septal lesion in vivo.

The control lesion experiments discussed above suggest that increased neurite-promoting activity in the hippocampal formation following a medial septal lesion is not simply a consequence of damage to nearby regions of the CNS (Nieto-Sampedro et al., 1983). Rather, it appears that damage to the septal innervation of the hippocampal formation produces the increase. It is not yet known whether damage to other hippocampal afferent pathways would also cause a similar increase in neurite-promoting activity. This is an important question since not all denervations of the hippocampal formation induce sympathetic ingrowth in vivo (Loy et al., 1980; Crutcher et al., 1981). The next logical extension of the present experiments, which we are currently pursuing, would be to determine the effect, on the level of NGF-like activity, of denervating lesions that do not induce sympathetic sprouting.

Although the NGF-like activity in hippocampal conditioned medium correlates in several ways with sympathetic sprouting in vivo, as discussed above, our results also indicate that hippocampal conditioned medium contains a small background of neurite-promoting activity that is insensitive to anti-NGF antibody (Fig. 1). We have found that the amount of this antibody-insensitive activity does not vary from region to region and is not affected by a medial septal lesion (Fig. 1). Based on these observations, we conclude that the antibody-insensitive activity is probably unrelated to sympathetic 


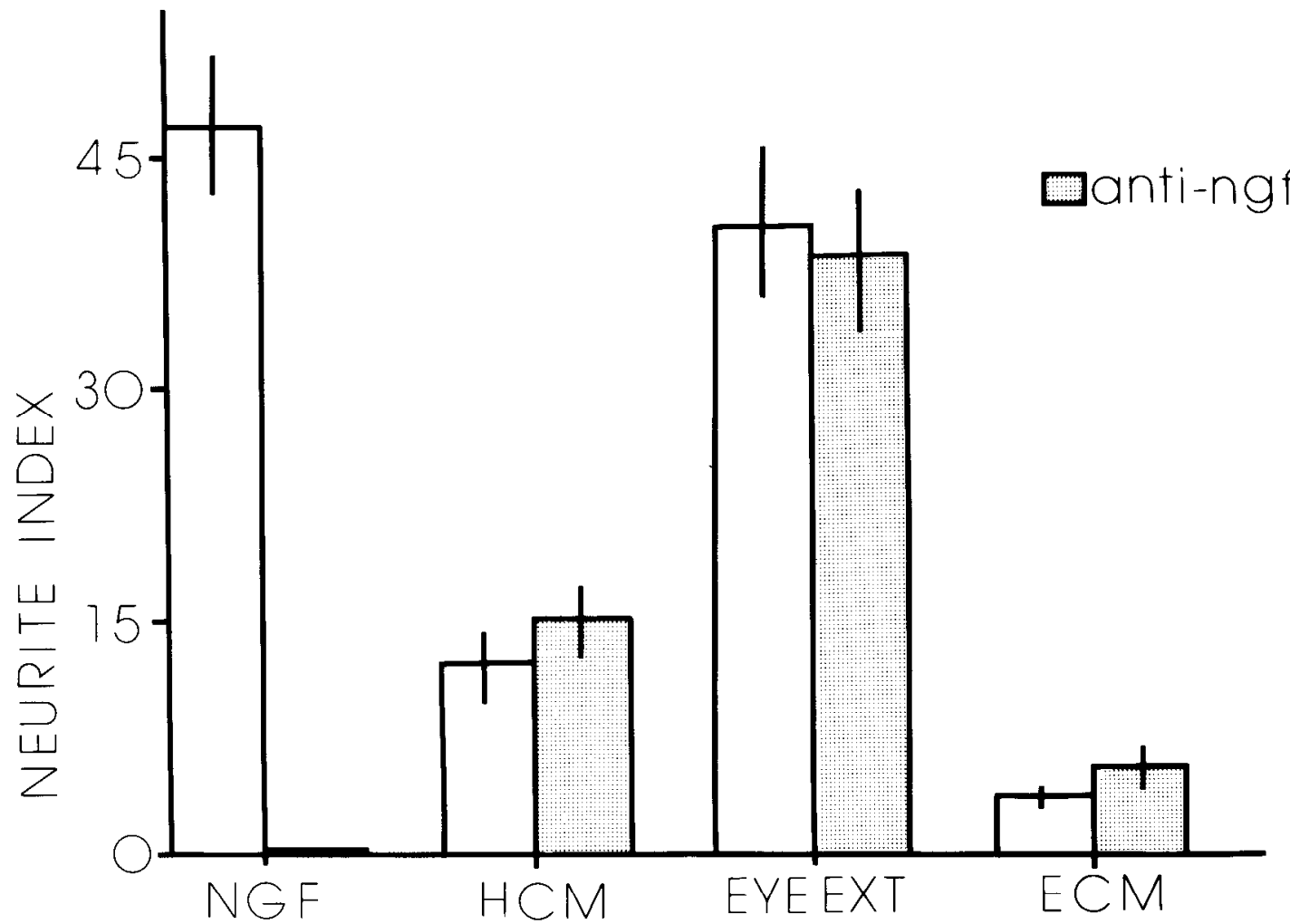

Figure 3. Effect of antibodies against $2.5 \mathrm{~S}$ NGF on the stimulation of sympathetic neurite growth by agents other than hippocampal conditioned medium. One of the following was added to standard cullure medium in order to stimulate sympathetic neurite growth: mouse submaxillary gland $\beta$-NGF at 2 ng/ml, chick embryo eye extract (EYE EXT), or conditioned medium from chick embryo heart cells (HCM) or mouse parietal yolk sac endoderm cells (ECM). Culture media were then divided into two aliquots, one of which was exposed to $5 \mu \mathrm{g} / \mathrm{ml}$ of anti-NGF (right bar of each pair of bars) and the other aliquot serving as an untreated control (left bar of each pair). Sympathetic chain pieces were then cultured in these media for 2 days before the extent of neurite growth (NEURITE INDEX) was determined. Each bar represents the mean neurite index \pm the standard deviation for two separate experiments. There was no statistically significant difference between heart conditioned medium, parietal yolk sac conditioned medium, and eye extract treated with antibody or without $(p>0.25)$

sprouting. A low level of anti-NGF-insensitive nerve growth activity has also been reported in brain tissues by others (Nieto-Sampedro et al., 1983).

In previous studies, the proposal was made that the granule cells and/or their axons (mossy fibers) were the most likely candidates for the source of the putative NGF-like growth factor. This conclusion was based on the co-distribution of sympathetic fibers with the granule cells and mossy fibers in dentate-CA3 and the observation that destruction of the granule cells prevented sympathetic ingrowth after a medial septal lesion (Crutcher and Davis, 1982). The significantly higher levels of activity in medium conditioned by dentateCA3 compared to CA1 is consistent with this proposal. However, there was detectable NGF-like activity in conditioned medium from the CA1 region, and the level of CA1 activity increased significantly 7 days after a medial septal lesion (Fig. 1). This observation was unexpected, since CA1 contains no granule cells or mossy fibers. There are several possible explanations for this observation. One is that we inadvertently included some dentate-CA3 tissue in the CA1 slices used to prepare conditioned medium. We did find several cases in which such contamination had occurred. However, the amount of dentate-CA3 tissue included in CA1 was always very small, and it seems unlikely that this could account for the activity observed in CA1. Another possibility is that the activity in CA1 diffused there from dentate-CA3. The likelihood of this is difficult to estimate. The final possibility is that the NGF-like activity in CA1 was generated by elements intrinsic to CA1, which excludes the granule cells and mossy fibers. Whatever the reason for NGF-like growth activity being present in $\mathrm{CA} 1$, it is still reasonable to conclude that the restriction of sympathetic fibers to dentate-CA3 in vivo may be correlated with the higher levels of NGF-like activity in dentate-CA3 after a medial septal lesion compared to CA1 and the fact that these increased levels are maintained for at least 4 weeks, which is the time it takes for the sympathetic ingrowth to become fully established, whereas by 4 weeks the levels in CA1 have decreased to those of unlesioned animals (Fig. 2).

Our results suggest that the stimulus to sympathetic sprouting after lesion may come from the sustained increase in the concentration of NGF-like growth activity above the levels present in the dentate-CA3 region of unlesioned animals. However, it is not possible from our experiments to determine the concentration of this agent in the normal unlesioned animal, since removal of the hippocampal formation to produce conditioned medium for 2 days in culture also causes denervation. Therefore, the levels of activity in our unlesioned animals may actually represent those appropriate to a 2-day denervation, whereas the levels in lesioned animals are representative of a 9 - to 30 -day denervation. Thus, we can only state from our results that the NGF-like activity increases substantially between 2 and 9 days after denervation in the dentate-CA3 and Imaintains this increased level for up to 4 weeks.

The increased NGF-like activity observed after a medial septal lesion indicates that the availability of the growth factor is regulated by the septohippocampal innervation. Since the septohippocampal neurons exhibit specific uptake and retrograde transport of NGF (Schwab et al., 1979), it is possible that the septal fibers normally act as a sink to remove this agent by uptake followed by retrograde transport or destruction. However, other possibilities include increased production of the factor after denervation or increased release due to cell damage. Whatever the underlying cause of the 
increase in activity due to denervation, it is presumably the increase in the amount of growth-promoting activity above a certain threshold after a medial septal lesion that triggers sympathetic sprouting.

\section{References}

Adler, R., K. B. Landa, M. Manthorpe, and S. Varon (1979) Cholinergic neuronotrophic factors. II. Selective intraocular distribution of soluble trophic activity for ciliary ganglionic neurons. Science 204: 1434-1436.

Berg, D. K. (1984) New neuronal growth factors. Annu. Rev. Neurosci. 7 149-170.

Bjorklund, A., and U. Stenevi (1981) In vivo evidence for a hippocampal neurotrophic factor specifically released on septal deafferentation. Brain Res. 229: 403-428.

Collins, F. (1978a) Axon initiation by ciliary neurons in culture. Dev. Biol. 65 : $50-57$.

Collins, F. (1978b) Induction of neurite growth by a conditioned medium factor bound to the culture substratum. Proc. Natl. Acad. Sci. U. S. A. 75: 5210-5213.

Collins, F. (1984a) Multiple sites for the regulation of neurite outgrowth. In Cellular and Molecular Biology of Neuronal Development, I. Black, ed., pp. 217-230, Plenum Press, New York.

Collins, F. (1984b) An effect of nerve growth factor on the parasympathetic ciliary ganglion. J. Neurosci. 4: 1281-1288.

Collins, F., and A. Dawson (1983) An effect of nerve growth factor on parasympathetic neurite outgrowth. Proc. Natl. Acad. Sci. U. S. A. 80 . 2091-2094.

Crutcher, K. A., and F. Collins (1982) In vitro evidence for two distinct hippocampal growth factors: Basis of neuronal plasticity? Science 217 $67-68$.

Crutcher, K. A., and J. N. Davis (1981) Sympathohippocampal sprouting is directed by a target tropic factor. Brain Res. 204: 410-414.

Crutcher, K. A., and J. N. Davis (1982) Target regulation of sympathetic sprouting in the rat hippocampal formation. Exp. Neurol. 75: 347-359.

Crutcher, K. A., L. Brothers, and J. N. Davis (1979) Sprouting of syrnpathetic nerves in the absence of afferent input. Exp. Neurol. 66: 778-783.
Crutcher, K. A., L. Brothers, and J. N. Davis (1981) Sympathetic noradrenergic sprouting in response to central cholinergic denervation: $A$ histochemical study of neuronal sprouting in the rat hippocampal formation. Brain Res. 210: 115-128.

Gage, F. H., A. Bjorklund, and U. Stenevi (1984) Denervation releases a neuronal survival factor in adult rat hippocampus. Nature 308: 637-639.

Helfand, S. L., R. J. Riopelle, and N. K. Wessells (1978) Non-equivalence of conditioned medium and NGF for sympathetic, parasympathetic and sensory neurons. Exp. Cell Res. 113: 39-45.

Lehman, J. M., W. C. Speers, D. E. Schwarzendruber, and G. B. Pierce (1974) Neoplastic differentiation: Characteristics of cell lines derived from murine teratocarcinoma. J. Cell. Physiol. 84: 13-28.

Levi-Montalcini, R., M. G. Menesini Chen, and J. S. Chen (1978) Neurotropic effects of the nerve growth factor in chick embryos and neonatal rats. Zoon 6: 201-212.

Loy, R., and R. Y. Moore (1977) Anomalous innervation of the hippocampal formation by peripheral sympathetic axons following mechanical injury. Exp. Neurol. 57: $64 b-650$.

Loy, R., T. A. Milner, and R. Y. Moore (1980) Sprouting of sympathetic axons in the hippocampal formation: Conditions necessary to elicit ingrowth. Exp. Neurol. 67: 399-411.

Milner, T. A., and R. Loy (1980) A delayed sprouting response to partial hippocampal deafferentation: Time course of sympathetic ingrowth following fimbrial lesions. Brain Res. 197: 391-399.

Nieto-Sampedro, M., M. Manthorpe, G. Barbin, S. Varon, and C. W. Cotman (1983) Injury-induced neuronotrophic activity in adult rat brain: Correlation with survival of delayed implants in the wound cavity. J. Neurosci. 3 . 2219-2229.

Schwab, M. E., U. Otten, Y. Agid, and H. Thoenen (1979) Nerve growth factor (NGF) in the rat CNS: Absence of specific retrograde axona transport and tyrosine hydroxylase induction in locus coeruleus and substantia nigra. Brain Res. 168: 473-483.

Stenevi, U., and A. Bjorklund (1978) Growth of vascular sympathetic axons into the hippocampus after lesion of the septo-hippocampal pathway: A pitfall in brain lesion studies. Neurosci. Lett. 7: 219-224. 